\title{
Genetic variation of the riparian pioneer tree species Populus nigra. II. Variation in susceptibility to the foliar rust Melampsora larici-populina
}

\author{
AGNÈS LEGIONNET*๋, HÉLÈNE MURANTY† \& FRANÇOIS LEFÈVRE \\ $\dagger$ INRA, Station d'Amélioration des Arbres Forestiers, 45160 Ardon, France and $\ddagger$ INRA, Unité de Recherches \\ Forestières Méditerranéennes, Avenue Vivaldi, 84000 Avignon, France
}

\begin{abstract}
Partial resistance of Populus nigra L. to three races of the foliar rust Melampsora larici-populina Kleb. was studied in a field trial and in laboratory tests, using a collection of $P$. nigra originating from different places throughout France. No total resistance was found. The partial resistance was split into epidemiological components, which proved to be under genetic control. Various patterns of association of epidemiological components values were found. Principal components analysis revealed their relationships. Only $24 \%$ of the variance of the field susceptibility could be explained by the variation of the epidemiological components of susceptibility. This variable was significantly correlated with susceptibility to the most ancient and widespread race of the pathogen, and with the variables related to the size of the lesions of the different races. Analysis of variance showed significant differences in susceptibility between regions and between stands within one region. Up to $20 \%$ of variation was between regions, and up to $22 \%$ between stands, so that these genetic factors appeared to be more differentiated than the neutral diversity (up to 3.5\%; Legionnet \& Lefèvre, 1996). However, no clear pattern of geographical distribution of diversity was detected.
\end{abstract}

Keywords: differentiation, genetic diversity, genetic resources, Melampsora larici-populina, Populus nigra, quantitative variation.

\section{Introduction}

The European black poplar Populus nigra L. is a common pioneer species of the riparian forest in Europe and western Asia. This species plays an important role in the world poplar wood production, mainly as a parent of interspecific hybrid clones involving $P$. deltoides. The foliar rusts Melampsora larici-populina Kleb. and M. allii-populina Kleb. belong to the natural pathogenic environment of both native P. nigra and cultivated poplars (Pinon, 1992). These fungi cause a reduction in the efficiency of photosynthesis and early defoliation in summer, and can lead to the death of the most susceptible clones. Poplar breeding has led to the cultivation of resistant hybrid clones, in which genes for

*Correspondence and present address: Université de Rennes I, Laboratoire de Botanique, 35042 Rennes Cedex, France. E-mail: agnes.schermann@univ-rennes1.fr resistance were presumably introduced from $P$. deltoides (Lefèvre et al., 1994). But the rapid evolution of $M$. larici-populina has led to the emergence of several races of this pathogen that have overcome these specific resistances through specific virulences (Pinon et al., 1987; Pinon \& Peulon, 1989). The most ancient race, E1, has no specific virulence. The races E2 and E3, which appeared, respectively, in northern Europe in 1982 (Pinon et al., 1987) and in Italy in 1989 (Pinon \& Peulon, 1989) are able to infect poplar clones resistant to E1. They rapidly spread in the regions where poplar clones resistant only to E1 were cultivated. Other new races have recently been described (Pinon \& Lefèvre, 1995; Steenackers et al., 1995). However, no specific resistance has been found in P. nigra, even though the susceptibility to $M$. larici-populina shows quantitative variation. Natural selection pressure caused by rusts may be very strong, especially for young individuals when competition is important (Legionnet, 1996), as has 
been shown in the related species Salix viminalis and Melampsora epitea (Verwijst, 1993). With natural infection by $M$. larici-populina and M. allii-populina, Cellerino et al. (1986) showed significant differences in infection levels between regions of provenance of Italian genotypes, and attributed these differences to geographically differentiated natural selection pressures. Furthermore, Pinon (1995) showed that the mix of rust races found in natural stands of $P$. nigra was influenced by the local presence of resistant cultivated clones. In this work, which is part of a survey of genetic resources of $P$. nigra that also includes a study of allozyme variation (Legionnet \& Lefèvre, 1996), the variation of traits related to the susceptibility to $M$. larici-populina is described. The relationship between different epidemiological components of resistance is studied, as well as the geographical organization of diversity in France, using natural and controlled conditions of inoculation and growth of the fungus.

\section{Materials and methods}

This study was carried out on a stool-bed collection of individual trees held by INRA in Orléans. The majority of genotypes were randomly sampled throughout France over a period of 20 years (Teissier du Cros, 1977; H. Duval, O. Duval \& F. Lefèvre, unpubl. data). The sample used comprised a total of 286 individuals originating from different sites (Fig. 1). Although vegetative propagation occurs, this study has shown that this is rare enough during the establishment of natural stands for the risk of sampling several vegetative copies of the same genotype to be very low when sampling adult trees (Legionnet et al., 1997). For each collection site, latitude, longitude and altitude were noted, as well as meteorological characteristics, monitored at the nearest meteorological station: mean annual rainfall, mean annual rainfall during the period that the trees are in leaf, mean annual lowest temperature, mean annual highest temperature and mean annual number of days of frost, over the last 30 years. Nine different regions were represented. Within one region (the Loire river valley), three additional stands were more densely sampled and included in the experiment: Les Brocs $\left(47^{\circ} 28^{\prime} \mathrm{N}, 2^{\circ} 54^{\prime} \mathrm{E}\right.$, 13 individuals), Ilot des Loges $\left(47^{\circ} 15^{\prime} \mathrm{N}, 2^{\circ} 58^{\prime} \mathrm{E}, 17\right.$ individuals) and Tinte $\left(46^{\circ} 50^{\prime} \mathrm{N}, 3^{\circ} 23^{\prime} \mathrm{E}\right)$. This last stand was subdivided into four zones corresponding to age classes (Legionnet, 1996), and these were represented by 51, 15, 19 and 14 randomly sampled individuals. Thus, two hierarchical levels were available for the geographical analysis: (i) among regions within France, some of which included several river systems separated by mountains (Alps, Rhône, Pyrénées), whereas others included only one river system (Durance: one river in the southern Alps; Loire: open and lowland river system of Loire and Allier); (ii) among stands within the Loire region (stands at Les Brocs and Ilot des Loges and the four zones of the stand at Tinte).

The field susceptibility and seven epidemiological components of susceptibility were studied here, as

Fig. 1 Map of the different accessions of the

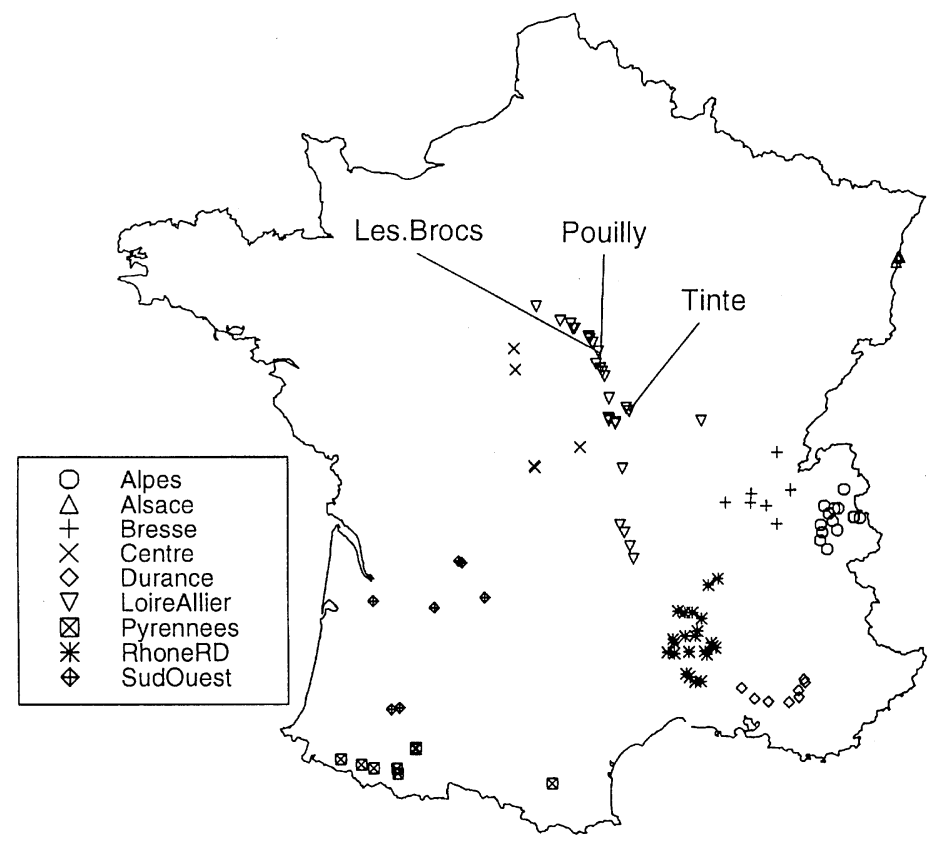
study collection of Populus nigra, and their distribution within regions and stands.

(C) The Genetical Society of Great Britain, Heredity, 82, 318-327. 
proposed by Lefèvre et al. $(1994,1995)$ : latent period for the race E1, number of uredia formed during one cycle of the pathogen for the races E1, E2 and E3, and number of urediospores per uredium for the races E1, E2 and E3. The field susceptibility was measured in a field trial: cuttings were obtained from each tree, and a randomized complete-block experiment, with six replications per genotype, was set up in the nursery at INRA, Orléans in 1995. The impact of rust on leaves was scored between 8 and 11 September 1995 following a nine-class scale, which took into account the number of infected leaves and the spread of the fungus on the infected leaves (category 1 was when no rust was observed anywhere on the plant, and category 9 was when more than $75 \%$ of the surface of the lamina was covered by rust on more than $25 \%$ of the leaves of the plant). The infection was natural, and it was not possible to distinguish between rust species or races. However, the annual survey of the rust population in this nursery has always revealed a high frequency of race E1 of $M$. laricipopulina (Pinon, 1995, pers. comm.). The other study variables were evaluated in the laboratory on one single cycle of the pathogen. One laboratory test was carried out for each of the three races E1, E2 and E3. Singlerace isolates propagated on differential clones were kindly provided by Dr Pinon (INRA, Nancy), and were stored at $4^{\circ} \mathrm{C}$ until used in tests. Inocula were prepared by suspension of spores at a concentration of 5000$10000 \mathrm{~mL}^{-1}$ in distilled water with $2 \%$ agar. The percentage germination of spores was verified to be more than $60 \%$ for each inoculum; this ensures that comparisons between the different tests are possible (J. Pinon, pers. comm.). The 286 individual tree genotypes were tested in each test. Leaves located between the fifth and tenth leaf from the apex on the main shoot of plants grown from cuttings in a greenhouse were collected, and four discs of $2 \mathrm{~cm}$ diameter were cut out of the lamina. The leaf discs were laid abaxial side up on wet tissue paper in Petri dishes. Twenty-five places were available in each dish, and one place in each dish was used for a foliar disc of the control clone 'Robusta'. Each test comprised four complete replications. For each replication, the 286 foliar discs to be tested were distributed following an incomplete block design into 13 dishes. The four replications (52 dishes) of the test for each isolate race were sprayed simultaneously with the inoculum, then the closed dishes were laid in a room with controlled temperature $\left(19^{\circ} \mathrm{C}\right)$ and lighting $(16 \mathrm{~h}$ day). Fifteen days after inoculation (i.e. after one multiplication cycle) the total number of uredia per leaf disc was counted, and the mean size of uredia was compared to the mean size of uredia of the control within the dish. For the test involving the race E1, the number of uredia on each disc was also scored 8 days after inoculation.

The study variables derived from these tests were: (i) lo1, lo2 and lo3, variables related to the number of uredia after one cycle of the pathogen for the races E1, E2 and E3, respectively; the variable is the logarithm of the number of uredia per disc (the logarithmic transformation was used to normalize the distribution of residuals in the ANOVA); (ii) si1, si2 and si3, variables related to the number of urediospores per uredium, and hence to the size of uredia, for the races E1, E2 and E3, respectively; a score with three classes was used for this variable (categories 1, 2 and 3 were given for uredia smaller than, equal to, or larger than uredia on 'Robusta', respectively); and (iii) la1, a variable related to the latent period (time by which half the final number of uredia have appeared) for the race E1; a score with four classes was used for this variable, based on the comparison between the two scores of the number of uredia measured at 8 and 15 days after inoculation (1, no uredium 1 week after inoculation; 2 , between one uredium and half the final number; 3 , more than half the final number, but fewer than the final number; 4, maximum number reached 1 week after inoculation. For all variables, high values correspond to higher susceptibility.).

Data analysis was performed with the s-PLus computer package (version 3.2, Statistical Sciences Inc., Seattle 1991) and software MODLI (Kobilinsky, 1990), specialized for the linear model, allowing for imbalance. ANOVA was used with the model (1) for the seven variables measured in the laboratory tests (hereafter referred to as laboratory variables):

$$
\begin{aligned}
\text { variable }= & \mu+\text { replic }^{\prime}+\text { dish in }_{\text {replic }} \\
& + \text { clone }+\varepsilon
\end{aligned}
$$

where $\mu$ is the general mean, replic' is the effect of the replication, dish in replic' is the effect of the dish (incomplete block) in each of the four complete replications, $\varepsilon$ is the residual (it contains no genetic variance) and the clone effect is considered as random. Graphical examination of the residuals did not show any departure from the assumptions required for the ANOVA.

The proportion of nongenetic variance controlled by the experimental design varied from $11 \%$ (si2, size of uredia, race E2) to $26 \%$ (lo2, number of uredia, race E2). In order to simplify the models used henceforth, the analyses use values of the variables adjusted for experimental environmental variation computed after this first analysis. 
For the field susceptibility, ANOVA was performed with the following mixed models ( 2 and 3 ), where the clone effect is considered as random, and the region and stand effects are considered as fixed:

$$
\begin{aligned}
\text { variable }= & \mu+\text { block }+ \text { region } \\
& + \text { clone in region }+\varepsilon \\
\text { variable }= & \mu+\text { block }+ \text { stand } \\
& + \text { clone in stand }+\varepsilon .
\end{aligned}
$$

The proportion of nongenetic variance controlled by the experimental design was $41 \%$.

For all variables, the broad-sense clonal heritabilities $\left(h_{\mathrm{sl}}^{2}\right)$ were calculated as follows: $h_{\mathrm{sl}}^{2}=\sigma_{\mathrm{G}}^{2} /\left[\sigma_{\mathrm{G}}^{2}+\sigma_{\mathrm{E}}^{2}\right]$ where $\sigma_{\mathrm{G}}^{2}$ is the proportion of variance attributable to the genetic factors [clone in model (1), region + clone in region in model (2)], and $\sigma_{\mathrm{E}}^{2}$ the proportion of nongenetic variance. The geographical structure of genetic variation was assessed using mixed models (2-5), where the clone effect was considered as random.

For the nine regions:

variable $=\mu+$ region + clone in region $+\varepsilon$.

For the stands in the Loire river region:

variable $=\mu+$ stand + clone in stand $+\varepsilon$.

The genotypic correlations between the laboratory variables were computed using the adjusted clonal means. Linear regressions of these variables were also performed on the geographical data (latitude, longitude and altitude of the site of origin), as well as multiple linear regressions on the geographical variables alone, and on both the geographical and meteorological variables. Principal components analysis (PCA) of normalized adjusted clonal means for the laboratory variables was performed, the field resistance being compared to the other variables as a supplementary variable. For each clone, the different adjusted clonal means of laboratory variables, expressed as percentages of the general means, allowed definition of a characteristic pattern of susceptibility.

\section{Results}

In the following, as we are dealing with many variables and thus performing numerous statistical tests, we will only consider 0.01 as a reliable rejection level in order to lower the global risk of type I error.

\section{Genetic control of the resistance components}

The characteristics of the different variables are summarized in Table 1. In the models (1) and (2), significant clone effects $(P<0.01)$ showed that the eight study variables were under genetic control. The broad-sense heritabilities revealed medium (0.26) to strong (0.59) genetic control. The laboratory variables related to the same race display moderate positive correlations with each other $(0.17-0.37 ; 0.01<P<0.05)$. Between races, only the variables measuring the same parameters for the races E2 and E3 displayed moderate correlations ( 0.40 for both size (si) and number of uredia (lo); $0.01<P<0.05$ ).

The correlations between the laboratory variables and the field susceptibility are shown in Table 2, as well as the correlations between the laboratory variables and the geographical and meteorological data. The field susceptibility was weakly correlated $(0.01<P<0.05)$ with the laboratory components related to the size of uredia (si1, si2 and si3) and with the number of uredia for the race E1 (lo1).

The PCA defined four principal axes representing $78.1 \%$ of the total variation. The correlation circles representing the variables are shown in Fig. 2(a). The first principal axis $(28.8 \%$ of the total variation) accounted for general susceptibility: all variables were represented on this axis, and had positive coordinates.

Table 1 Characterization of the eight variables measured on the Populus nigra collection

\begin{tabular}{lcccccccc}
\hline & lo1 & la1 & sil & lo2 & si2 & lo3 & si3 & field \\
\hline Range & $0-3$ & $0-4$ & $0.5-3$ & $0-4$ & $1-3$ & $0-3$ & $1-3$ & $4-9$ \\
Mean value on P. nigra & 1.48 & 2.25 & 1.73 & 1.82 & 1.97 & 1.74 & 1.88 & 6.09 \\
Mean value on 'Robusta' & 2.11 & 2.50 & 2 & 2.47 & 2 & 2.90 & 2 & 6.00 \\
Broad-sense heritability & 0.48 & 0.26 & 0.30 & 0.59 & 0.41 & 0.46 & 0.43 & 0.39 \\
$\sigma_{\mathrm{rg}}^{2}\left(\sigma_{\mathrm{rg}}^{2}+\sigma^{2} \mathrm{cl} / \mathrm{rg}\right)$ & $16 \%$ & $3 \%$ & $10 \%$ & $17 \%$ & $2 \%$ & $18 \%$ & $20 \%$ & $21 \%$ \\
$\sigma_{\mathrm{rt}}^{2}\left(\sigma_{\mathrm{rt}}+\sigma_{\mathrm{cl} / \mathrm{st}}\right)$ & $22 \%$ & $0.4 \%$ & $13 \%$ & $0.6 \%$ & $8 \%$ & $18 \%$ & $17 \%$ & $10 \%$ \\
\hline
\end{tabular}

la1, infectious latency for race E1; si1, si2 and si3, size of uredia for the races E1, E2 and E3; lo1, lo2 and lo3, logarithm of the number of uredia under laboratory conditions (see text) for the races E1, E2 and E3; field, field susceptibility; cl, clone; rg, region; st, stand.

(c) The Genetical Society of Great Britain, Heredity, 82, 318-327. 
Table 2 Correlations of the seven epidemiological components of susceptibility measured on the Populus nigra collection with field susceptibility and with geographical data, and percentage of variation of these variables explained by multiple regression $\left(R^{2}\right)$ of geographical $(G)$ and meteorological $(M)$ data on the variables

\begin{tabular}{lccccccc}
\hline & lo1 & la1 & si & lo2 & si2 & lo3 & si3 \\
\hline Field susceptibility & $0.154^{*}$ & NS & $0.368^{*}$ & NS & $0.276^{*}$ & NS & $0.265^{*}$ \\
Altitude & NS & NS & NS & NS & NS & NS & $* *$ \\
Longitude & $*$ & NS & NS & $* * *$ & NS & NS & NS \\
Latitude & NS & NS & $* * *$ & $* *$ & NS & $* *$ & $*$ \\
$R^{2}$ with G & $3.4 \% *$ & NS & $5.4 \% * *$ & $7.3 \% * * *$ & NS & $5.3 \% * *$ & $4.3 \% * *$ \\
$R^{2}$ with G and M & $16 \% * * *$ & NS & $7.1 \% * *$ & $11 \% * * *$ & NS & $8.6 \% * *$ & NS \\
\hline
\end{tabular}

la1, infectious latency for race E1; si1, si2 and si3, size of uredia for the races E1, E2 and E3; lo1, lo2 and lo3, logarithm of the number of uredia under laboratory conditions (see text) for the races E1, E2 and E3. $* P<0.05, * * P<0.01, * * * P<0.001$.
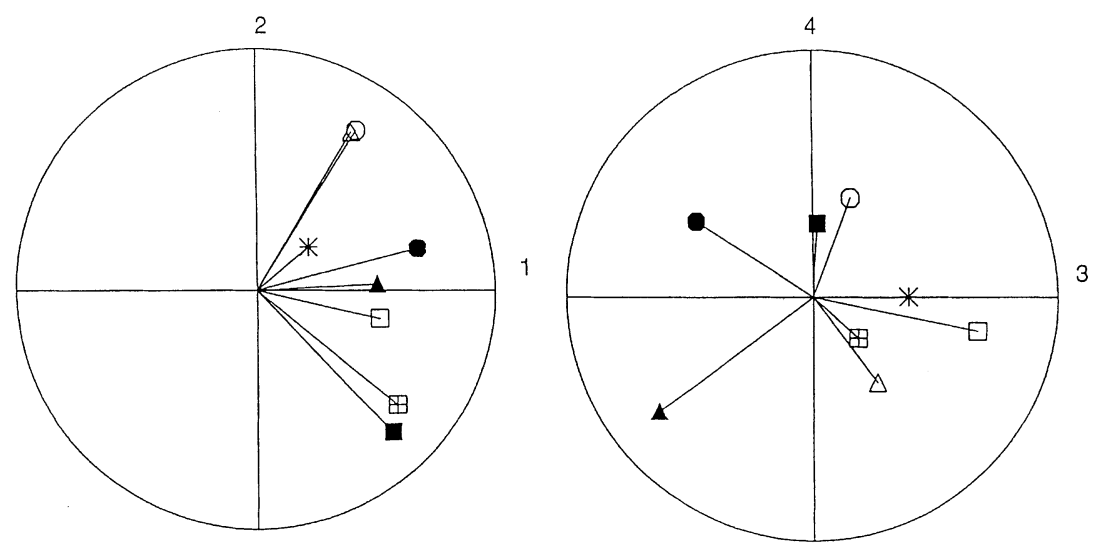

(a)
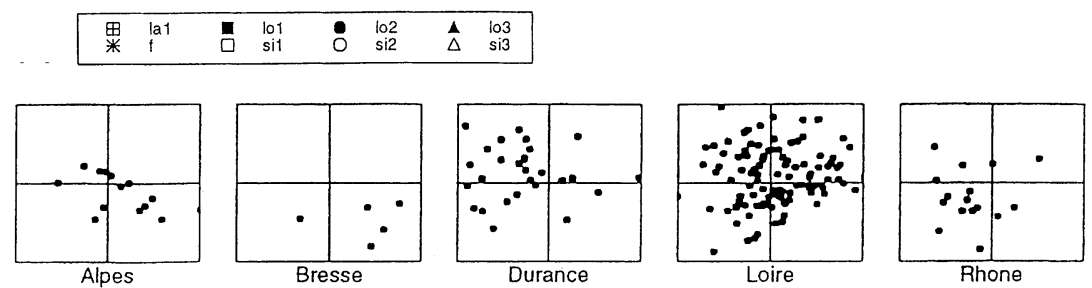

Fig. 2 Principal components analysis (PCA) of the eight variables related to the susceptibility of Populus nigra to Melampsora larici-populina. la1, infectious latency for race E1; si1, si2 and si3, size of uredia for the races E1, E2 and
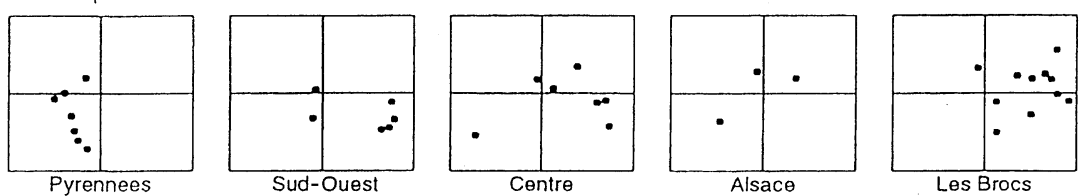

E3; lo1, lo2 and lo3, logarithm of the number of uredia under laboratory conditions (see text) for the races E1, E2 and E3; f, field susceptibility (complementary variable). (a) Correlations of the vari-
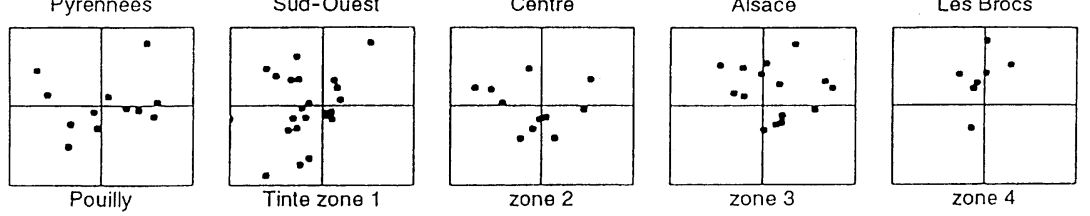
ables with the principal axes 1 and 2 (left) and 3 and 4 (right). (b) Positions of points representing the individuals of the different regions and stands on the plane defined by the principal axes 1 and 2 .

The second principal axis (21.5\%) accounted for the nature of resistance: resistance to E2 and E3 through smaller uredia (si2 and si3) was opposed to resistance to E1 through longer latency and a smaller number of uredia (la1 and lo1). Although the other variables were poorly represented on this axis, it should be noted that all the variables related to E1 were opposed to all the variables related to E2 and E3. The third principal axis $(17.3 \%)$ also accounted for the nature of resistance: the variables 102 and lo3 (number of uredia for races E2 and E3) were well represented on this axis (respectively $23 \%$ and $40 \%$ ), and were opposed to the variables si1, si2 and si3 (size of uredia for the three races); the variable sil was particularly well represented on this axis (46\%). All 
variables were almost equally represented on the fourth principal axis (10.5\% of total variation), and the variables related to E2 were opposed to the variables related to E3.

The diversity of individual susceptibility patterns over the seven laboratory variables was illustrated using the 10 individuals displaying the lowest field susceptibility (Fig. 3). These individuals, although displaying similar global performances, show varying susceptibility patterns.

\section{Geographical distribution of variation}

The analyses of variance revealed significant regional effects (Tables 3 and 4), but no clear pattern of geographical differentiation was revealed. The distribution of variation was not the same for all variables
(Table 1): the variance among regions ranged from $3 \%$ (la1, latency of E1) to $21 \%$ (field susceptibility) of the total genetic variance, and within the Loire region the variance between stands ranged from $0.4 \%$ (la1, latency of the race E1) to $22 \%$ (lo1, number of uredia for the race E1). Some regressions of the variables on the geographical data were found to be significant $(P<0.01)$ (Table 2). However, the multiple- $R^{2}$ of multiple regression reached only $7.3 \%$ with the geographical data, and $16 \%$ with the geographical and meteorological data. The distribution of individuals on the PCA planes (Fig. 2b) helps us to describe the pattern of geographical distribution of susceptibility: the Durance, Rhône and Pyrénées regions and the Tinte zone 1 stand are mostly located on the left part of the first factorial plane, which means that the global susceptibility is low in these groups, whereas it is high in the

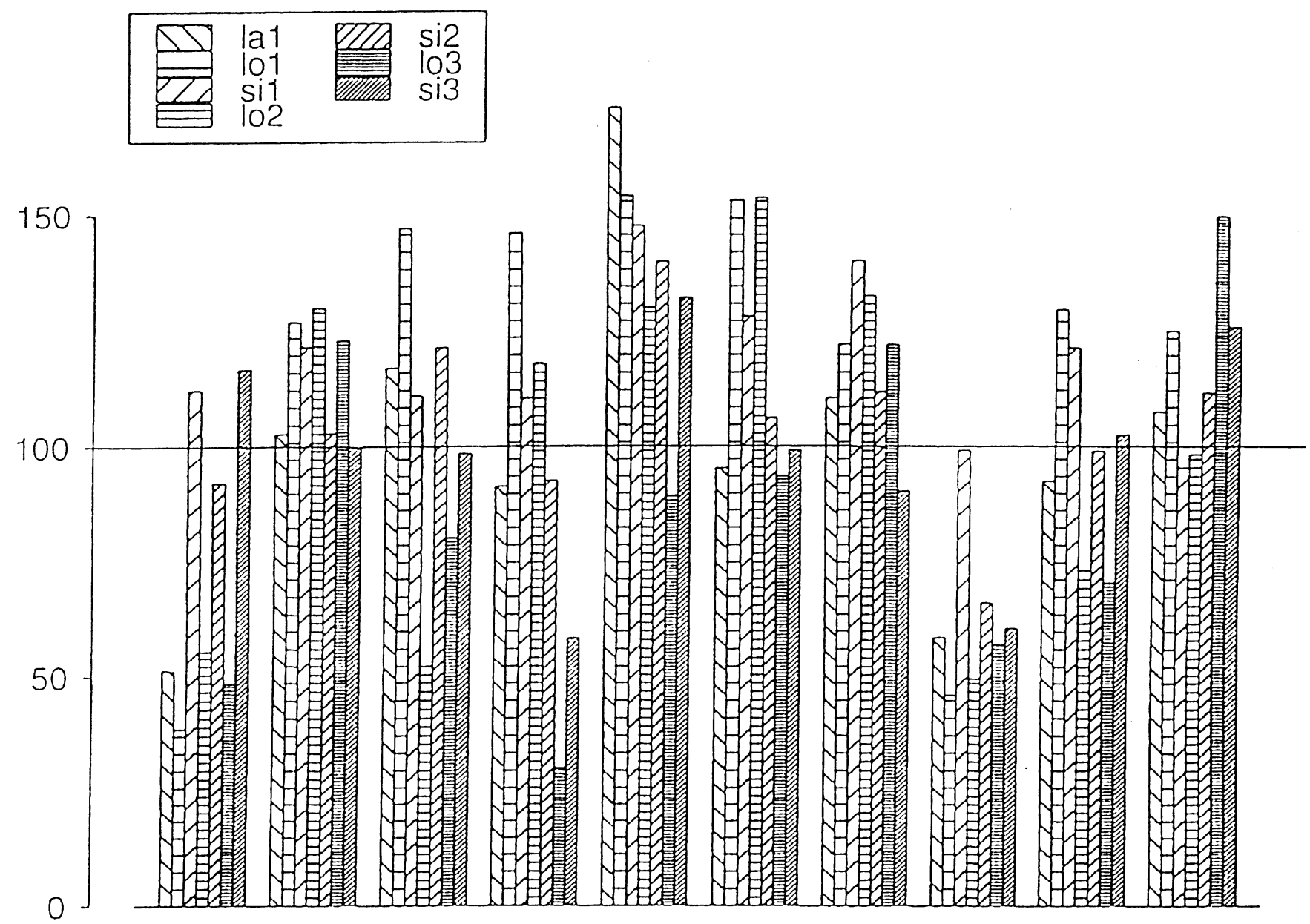

Fig. 3 Individual patterns of susceptibility of Populus nigra to Melampsora larici-populina for the seven laboratory variables, expressed in percentages of the mean value of the whole collection, for the 10 less susceptible $P$. nigra trees according to the field susceptibility. la1, infectious latency for race E1; si1, si2 and si3, size of uredia for the races E1, E2 and E3; lo1, lo2 and lo3, logarithm of the number of uredia under laboratory conditions (see text) for the races E1, E2 and E3; field, field susceptibility; cl, clone; rg, region; st, stand.

(C) The Genetical Society of Great Britain, Heredity, 82, 318-327. 
Table 3 Analysis of variance of the field susceptibility of Populus nigra to Melampsora larici-populina (variable 'field') with two models accounting for the repartition of individuals in blocks in the field trial, and, respectively, in regions and stands within one of the regions: (2) variable $=\mu+$ block + region + clone in region $+\varepsilon$; and (3) variable $=\mu+$ block + stand + clone in stand $+\varepsilon$

\begin{tabular}{|c|c|c|c|c|c|}
\hline Source & d.f. & SS & MS & $F$ & $P(\%)$ \\
\hline \multicolumn{6}{|l|}{ Model (2) } \\
\hline Block & 5 & 520.7 & 104.141 & 163.2 & 0.00 \\
\hline Region & 8 & 210.5 & 26.316 & 9.8 & 0.00 \\
\hline Clone in region & 264 & 708.2 & 2.682 & 4.2 & 0.00 \\
\hline Residual & 1155 & 736.9 & 0.638 & & \\
\hline Total & 1432 & 2176.0 & 1.520 & & \\
\hline \multicolumn{6}{|l|}{ Model (3) } \\
\hline Block & 5 & 299.2 & 59.834 & 87.5 & 0.00 \\
\hline Stand & 5 & 30.2 & 6.051 & 2.0 & 0.08 \\
\hline Clone in stand & 96 & 289.0 & 3.011 & 4.4 & 0.00 \\
\hline Residual & 453 & 309.8 & 0.684 & & \\
\hline Total & 559 & 928.3 & 1.661 & & \\
\hline
\end{tabular}

Bresse and Centre regions and in the Les Brocs stand. In the Alpes, Bresse, Rhône, Pyrénées and Sud-Ouest regions, most individuals were located on the lower part of this plane, which reveals a greater susceptibility to E1 than to E2 and E3, whereas in the Durance region and zones 3 and 4 of the Tinte stand, most individuals were less susceptible to E1.

\section{Discussion}

\section{Genetic diversity of rust resistance}

The epidemiological components of susceptibility studied here were shown to be under medium to strong genetic control. High heritability values have also been reported for traits related to rust susceptibility in Populus (Riemenschneider et al., 1994; P. balsamifera L.) and in Pinus (Ide-Souza et al., 1991). However, the field susceptibility was poorly explained by the laboratory variables (about $20 \%$ of the variation of the variable was represented on the different principal axes in the PCA). Furthermore, several of the most resistant individuals in the field displayed values lower than the general mean for almost all laboratory variables (Fig. 3). By way of contrast, on P. deltoides and Melampsora medusae, Hamelin et al. (1994) found significant correlations between the disease variables measured on leaf disc assays and the field susceptibility. But their study involved a small number of genotypes, among which they described one exception that was resistant in leaf disc assays but susceptible in the field trials. They pointed out that the reliability of the monocyclic disease parameters has been proved in different pathosystems, but the best predictor of field susceptibility varies from one pathosystem to another. In the present study, the field susceptibility was represented best on the second principal axis, and therefore we can conclude that the mechanism of resistance (size of uredia vs. number of uredia) and the susceptibility to E1 vs. susceptibility to E2 and E3 account best for the field susceptibility. A high correlation between the size of uredia and the field resistance has also been described for Populus trichocarpa (Lefèvre et al., 1994). In addition, we suggest that some other variable traits, which are not directly related to the development of the pathogen on the leaves, may also account for susceptibility in the field: date of the first infection, height and architecture of trees, and arrangement or mobility of leaves, which may play a role in limiting the infection in natural conditions. IdeSouza et al. (1991) showed independence between traits related to the development of the rust on leaves and traits related to the first infection in Pinus. In the present study, the independence between the epidemiological components of susceptibility was demonstrated by PCA: the susceptibility to E1 was opposed to the susceptibility to E2 and E3 on the second principal axis, and the susceptibility to E2 was opposed to the susceptibility to E3 on the fourth principal axis. In addition, for E2 and E3, the variables related to the size of uredia and the variables related to the number of uredia were represented on two separate axes. The existence of independent components of susceptibility is of great interest for breeding, because it shows the involvement of different genetic factors which could be combined in a single variety, thus leading to a low susceptibility that might be more difficult for the fungus to overcome. 
Table 4 Summary of the analysis of variance for the seven components of the susceptibility of Populus nigra to Melampsora larici-populina measured in laboratory tests, with two models accounting, respectively, for the repartition of individuals in regions and stands within one of the regions: (4) variable $=\mu+$ region + clone in region $+\varepsilon$; and (5) variable $=\mu+$ stand + clone in stand $+\varepsilon$

\begin{tabular}{|c|c|c|c|c|}
\hline Variable & & d.f. & $F$ & $P(\%)$ \\
\hline \multicolumn{5}{|l|}{ Model (4) } \\
\hline \multirow[t]{2}{*}{ lo1 } & $F($ region, clone in region $)$ & $8 ; 261$ & 5.48 & 0.00 \\
\hline & $F($ clone in region, residual $)$ & $261 ; 797$ & 4.1 & 0.00 \\
\hline \multirow[t]{2}{*}{ la1 } & $F($ region, clone in region $)$ & $8 ; 251$ & 1.52 & 14.9 \\
\hline & $F($ clone in region, residual $)$ & $251 ; 654$ & 2.2 & 0.00 \\
\hline \multirow[t]{2}{*}{ sil } & $F$ (region, clone in region) & $8 ; 251$ & 2.87 & 0.45 \\
\hline & $F$ (clone in region, residual $)$ & $251 ; 624$ & 2.3 & 0.00 \\
\hline \multirow[t]{2}{*}{102} & $F($ region, clone in region $)$ & $8 ; 255$ & 5.93 & 0.00 \\
\hline & $F($ clone in region, residual $)$ & $255 ; 763$ & 5.8 & 0.00 \\
\hline \multirow[t]{2}{*}{ si2 } & $F($ region, clone in region $)$ & $8 ; 247$ & 1.35 & 21.8 \\
\hline & $F$ (clone in region, residual $)$ & $247 ; 684$ & 3.5 & 0.00 \\
\hline \multirow[t]{2}{*}{ lo3 } & $F($ region, clone in region) & $8 ; 254$ & 5.59 & 0.00 \\
\hline & $F($ clone in region, residual $)$ & $254 ; 737$ & 3.6 & 0.00 \\
\hline \multirow[t]{2}{*}{ si3 } & $F($ region, clone in region $)$ & $8 ; 253$ & 6.07 & 0.00 \\
\hline & $F($ clone in region, residual $)$ & $253 ; 667$ & 3.1 & 0.00 \\
\hline \multicolumn{5}{|l|}{ Model (5) } \\
\hline \multirow[t]{2}{*}{ lo1 } & $F($ stand, clone in stand) & $5 ; 113$ & 5.15 & 0.03 \\
\hline & $F$ (clone in stand, residual) & $113 ; 340$ & 3.8 & 0.00 \\
\hline \multirow[t]{2}{*}{ la1 } & $F($ stand, clone in stand) & $5 ; 108$ & 1.05 & 39.4 \\
\hline & $F($ clone in stand, residual $)$ & $108 ; 268$ & 2.7 & 0.00 \\
\hline \multirow[t]{2}{*}{ sil } & $F($ stand, clone in stand) & $5 ; 108$ & 2.39 & 4.23 \\
\hline & $F$ (clone in stand, residual) & $108 ; 256$ & 2.0 & 0.00 \\
\hline \multirow[t]{2}{*}{ lo2 } & $F($ stand, clone in stand $)$ & $5 ; 111$ & 1.10 & 36.6 \\
\hline & $F$ (clone in stand, residual) & $111 ; 321$ & 5.5 & 0.00 \\
\hline \multirow[t]{2}{*}{ si2 } & $F($ stand, clone in stand $)$ & $5 ; 108$ & 2.33 & 4.71 \\
\hline & $F$ (clone in stand, residual) & $108 ; 295$ & 3.6 & 0.00 \\
\hline \multirow[t]{2}{*}{ lo3 } & $F($ stand, clone in stand $)$ & $5 ; 112$ & 3.88 & 0.28 \\
\hline & $F$ (clone in stand, residual $)$ & $112 ; 317$ & 3.2 & 0.00 \\
\hline \multirow[t]{2}{*}{ si3 } & $F($ stand, clone in stand) & $5 ; 112$ & 3.51 & 0.55 \\
\hline & $F$ (clone in stand, residual) & $112 ; 292$ & 2.6 & 0.00 \\
\hline
\end{tabular}

la1, infectious latency for race E1; si1, si2 and si3, size of uredia for the races E1, E2 and E3; lo1, lo2 and lo3, logarithm of the number of uredia under laboratory conditions (see text) for the races E1, E2 and E3.

\section{Geographical distribution of genetic diversity}

The existence of a geographical structure for the study variables was shown by the ANOVA, by the regression on the geographical data, and by the location of geographical groups on the planes of the PCA. This structure is not the same for all variables and is inadequately explained by the geographical data. Hence, the distribution of variation does not seem to be an effect of geographically structured natural selection, in contrast to the susceptibility of $P$. nigra to the woolly poplar aphid in Italy (Allegro \& Cagelli, 1996). In P. balsamifera, Riemenschneider et al. (1994) also described significant differences in susceptibility to rust between stands and between drainages, but the variation attributable to geographical structure was small relative to clonal variation, as is the case in the present study. The pattern of susceptibility found in the Alps region could be explained by artificial selection. Indeed, the individuals representing this region had been collected in 1971 and 1972 and submitted to weak artificial selection during 20 years in cultivation (the most infected individuals had been eliminated). Hence, a greater resistance to E1 was expected for this part of the collection. Indeed, the susceptibility was characterized by a rapid succession of cycles of the pathogen and the development of a great number of uredia; the small size of uredia leading, however, to a fair level of resistance. Artificial selection had favoured individuals with the smallest size of uredia of the race E1 (third principal axis), and the uredia size of the two other races appeared to be small also (second principal axis). The age of individuals could also explain the pattern of susceptibility, as shown in the Tinte stand. Indeed, for zones 3 and 4, where the individuals 
are the oldest of the stand, a low susceptibility to E1 was found: this distribution could result from natural selection caused by E1, which has been the most common race during the evolution of this stand. Unfortunately, age data of individuals sampled are not available for the whole collection.

\section{Consequences for resource management}

This collection displays some diversity for the studied traits, and for the association of these different traits. This diversity is well illustrated by the various individual profiles found for the trees displaying a low field susceptibility (Fig. 3). Weak but significant geographical differentiation was found. As shown in Fig. 2(b), the River Loire drainage system can be considered as representative of the present diversity in the different regions, hence a collection representative of the diversity of resistance components to rusts could be sampled in this region. However, it has been suggested in another study (Legionnet \& Lefèvre, 1996) that the low geographical differentiation in this species could be a relict of an ancient situation, when the species was more widespread, but that gene flow may not be sufficient nowadays to cause homogenization of regions. Therefore a collection sampled only in one region would not account for recent, different evolution of traits under different selection pressures. As shown by Pinon (1995), the racial composition of natural rust populations is changing rapidly nowadays, as illustrated by the recent expansion of races E2 and E3. Because of the age of the sampled trees, we can consider that the natural accessions included in our collection did not undergo selection by these new races, but the newly spread races will probably influence the further natural selection in P. nigra stands. Indeed, Pinon (1995) showed that the races E2 and E3 are very frequent only in the regions where poplar cultivation is practised, and the selection will therefore be geographically structured. As is the case for most natural pathogen populations (Burdon \& Jarosz, 1989), rust populations are also likely to undergo rapid changes in racial composition, caused by fluctuations in the nature of locally cultivated hybrid poplar clones. For these reasons, it is difficult to predict whether the changing selection pressure will induce differentiation between the natural $P$. nigra populations.

\section{Acknowledgements}

This work was supported by a grant from the Direction de l'Espace Rural et de la Forêt, Ministère de l'Agriculture et de la Forêt. We thank Daniel Lacan for help with laboratory tests and field measures, Dr C. Bastien and Dr N. Schermann for help with the statistical analysis, Professor Goffinet for verification of the validity of the data analysis, Dr J. Pinon for providing original inoculum of the different races and information about the pathogen, and the technical team that took care of the trees in the nursery. We thank the two anonymous reviewers for their useful comments on the first version of this manuscript.

\section{References}

Allegro, G. AND CAGElli, L. 1996. Susceptibility of Populus nigra $\mathrm{L}$. to the woolly poplar aphid (Phloeomyzus passerinii Sign.). Forest Genet. 3, 23-26.

BURDON, J. J. AND JAROSZ, A. M. 1989. Disease in mixed cultivars, composites, and natural plant populations: some epidemiological and evolutionary consequences. In: Brown, A. H. D., Clegg, M. T., Kahler, A. L. and Weir, B. S. (eds) Plant Population Genetics, Breeding, and Genetic Resources, pp. 215-228. Sinauer Associates, Sunderland, MA.

CELlerino, G. P., ANSElmi, N., BISOFFI, S., GIORCELlI, A. AND Belisario, A. 1986. Behaviour of Populus nigra L. coming from various sources towards Melampsora allii-populina Kleb. and Melampsora larici-populina Kleb. In: FAO International Poplar Commission, Working Party on Diseases, XXIV Conference, 22-24 September 1986. Bordeaux, France.

HAMELIN, R. C., FERRIS, R. S., SHAIN, L. AND THIELGES, B. A. 1994. Prediction of poplar leaf rust epidemics from a leaf-disk assay. Can. J. Forest Res., 24, 2085-2088.

IDE-SOUZA, S. M., WHITE, T. L., HODGE, G. R. AND SCHMIDT, R. A. 1991. Genetic parameter estimates for greenhouse traits of slash pine artificially inoculated with fusiform rust fungus. Forest Sci., 37, 836-848.

KOBILINSKY, A. 1990. MODLI, BAO Document no. 09/90, NCY/GL. Département d'Informatique, Institut National de la Recherche Agronomique. Versailles, France.

LEFÈVRE, F., PICHOT, C. AND PINON, J. 1994. Intra- and interspecific inheritance of some components of the resistance to leaf rust (Melampsora larici-populina Kleb.) in poplars. Theor. Appl. Genet., 88, 501-507.

Lefèvre, F., Chartier, S., FAivre-RAMPANT, P., GOUÉ, M. C., LAURENS, F., PILATE, G. ET AL. 1995. Composantes de la résistance aux rouilles chez les peupliers: utilisation en sélection. C. R. Acad. Agric. Fr., 81, 111-121.

Legionnet, A. 1996. Diversité et Fonctionnement Génétique des Populations Naturelles de Populus nigra L. Espèce Pionnière des Ripisylves Européennes. Thèse de doctorat, Université de Montpellier II, France.

LEGIONNET, A. AND LEFÈvRE, F. 1996. Genetic variation of the riparian tree species Populus nigra L. I. Study of population structure based on isozymes. Heredity, 77, 629-637.

LEGIONNET, A., FAIVRE-RAMPANT, P., VILLAR, M. and LEFÈVRE, F. 1997. Sexual and asexual reproduction in natural stands of Populus nigra. Bot. Acta, 110, 257-263.

PINON, J. 1992. Frequency and evolution of Melampsora laricipopulina Klebahn races in north-western France. Ann. Sci. For., 49, 1-15. 
PINON, J. 1995. Variability in poplar rusts and evolution of their populations. Consequences for control strategies. C. $R$. Acad. Agric. Fr., 81, 99-109.

PINON, J. AND LEFÈVRE, F. 1995. A new virulence found among isolates of Melampsora larici-populina. In: Frison, E., Lefèvre, F., De Vries, S. and Turok, J. (eds) Populus nigra Network, Report of the First Meeting, 3-5 October, 1994. Izmit, Turkey.

PINON, J. and PEUlON, v. 1989. Mise en évidence d'une troisième race physiologique de Melampsora laricipopulina Kleb. en Europe. Cryptogam. Mycol., 10, 95-106.

PINON, J., VAN DAM, B. C., GENETET, I. AND DE KAM, M. 1987. Two pathogenic races of Melampsora larici-populina in north-western Europe. Eur. J. For. Path., 17, 47-53.
RIEMENSCHNEIDER, D. E., MCMAHON, D. E. AND OSTRY, M. E. 1994. Population-dependent selection strategies needed for 2years-old black cottonwood clones. Can. J. Forest Res., 24, 1704-1710.

STEENACKers, M., STEENACKERS, V. and Delporte, T. 1995. A new physiological race of Melampsora larici-populina in Belgium. In: Frison, E., Lefèvre, F., De Vries, S. and Turok, J. (eds) Populus nigra Network, Report of the First Meeting, 3-5 October, 1994. Izmit, Turkey.

TEISSIER DU CROS, E. 1977. Aperçu de la transmission héréditaire de quelques caractères juvéniles chez Populus nigra L. Ann. Sci. For., 34, 311-322.

VERWIJST, T. 1993. Influence of the pathogen Melampsora epitea on intraspecific competition in a mixture of Salix viminalis clones. J. Vegetation Sci., 4, 717-722. 